\title{
Fotossíntese e acúmulo de solutos em feijoeiro caupi submetido à salinidade
}

\author{
Rogéria Pereira Souza(1), Eduardo Caruso Machado(2), Joaquim Albenísio Gomes Silveira(3) \\ e Rafael Vasconcelos Ribeiro ${ }^{(2)}$
}

\begin{abstract}
(1)In memoriam. (2)Instituto Agronômico, Centro de Pesquisa e Desenvolvimento em Ecofisiologia e Biofísica, Laboratório de Fisiologia Vegetal Coaracy M. Franco, Avenida Barão de Itapura, no 1.481, Botafogo, CEP 13012-970 Campinas, SP. E-mail: caruso@iac.sp.gov.br, rafael@iac.sp.gov.br (3)Universidade Federal do Ceará, Departamento de Bioquímica e Biologia Molecular, Caixa Postal 6020, CEP $60451-970$ Fortaleza, CE. E-mail: silveira@ufc.br
\end{abstract}

Resumo - O objetivo deste trabalho foi determinar as respostas fotossintéticas e a acumulação de carboidratos, íons salinos e prolina em feijoeiro caupi (Vigna unguiculata) submetido à salinidade. As plantas foram submetidas à quatro tratamentos, dos 28 aos 35 dias de idade: $0,50,100$ e $200 \mathrm{mmol} \mathrm{L}^{-1}$ de $\mathrm{NaCl}$. Avaliaramse as trocas gasosas, a emissão de fluorescência pela clorofila $a$, o potencial hídrico foliar, e as concentrações de carboidratos, $\mathrm{Na}^{+}, \mathrm{Cl}^{-}$e prolina nas folhas. Os tratamentos não tiveram efeito sobre a eficiência quântica potencial do fotossistema II, mas causaram leve diminuição na eficiência quântica efetiva e maior dissipação do excesso de energia de excitação por processos não fotoquímicos. As concentrações foliares de amido diminuíram, e as de sacarose e prolina aumentaram nas maiores concentrações de $\mathrm{NaCl}$. Ocorreu exclusão do $\mathrm{Na}^{+}$e acúmulo do $\mathrm{Cl}^{-}$nas folhas, e as relações hídricas das folhas foram pouco afetadas, exceto no tratamento mais severo. $\mathrm{O}$ acúmulo de $\mathrm{Cl}^{-}$esteve envolvido na redução da assimilação de $\mathrm{CO}_{2}$, decorrente da queda na condutância estomática e na eficiência de carboxilação da Rubisco. O feijoeiro caupi apresenta características fisiológicas que favorecem a manutenção da atividade fotossintética sob curta exposição à salinidade.

Termos para indexação: Vigna unguiculata, condutância estomática, eficiência de carboxilação, fluorescência da clorofila, prolina, trocas gasosas.

\section{Photosynthesis and accumulation of solutes in cowpea plants subjected to salinity}

\begin{abstract}
The objective of this work was to determine the photosynthetic responses and the accumulation of carbohydrates, salt ions, and proline in cowpea (Vigna unguiculata) plants subjected to salinity. Plants were subjected to four treatments, from 28 to 35 days of age: $0,50,100$, and $200 \mathrm{mmol} \mathrm{L}^{-1}$ of NaCl. Leaf gas exchange, chlorophyll $a$ fluorescence, leaf water potential, and leaf concentrations of carbohydrates, $\mathrm{Na}^{+}, \mathrm{Cl}^{-}$, and proline were evaluated. Treatments had no effect on potential quantum efficiency of photosystem II, but there was a slight reduction in effective quantum efficiency and a higher dissipation of excess excitation energy by non-photochemical processes. The foliar contents of starch decreased, and those of sucrose and proline increased with higher $\mathrm{NaCl}$ content. There was $\mathrm{Na}^{+}$exclusion and $\mathrm{Cl}^{-}$accumulation in leaves, and leaf water relations were little affected by salt stress, except in the most severe treatment. The accumulation of $\mathrm{Cl}^{-}$was related with the reduction of $\mathrm{CO}_{2}$ assimilation, which resulted from the decline in stomatal conductance and in Rubisco carboxylation efficiency. Cowpea plants have physiological characteristics that favor the maintenance of photosynthetic activity under short-term exposure to salinity.
\end{abstract}

Index terms: Vigna unguiculata, stomatal conductance, carboxylation efficiency, chlorophyll fluorescence, proline, gas exchange.

\section{Introdução}

A salinidade é um dos principais estresses ambientais que afetam negativamente o crescimento e o metabolismo vegetal, além de ser um dos fatores responsáveis pelo decréscimo da produtividade de culturas nas regiões áridas e semiáridas. A redução do crescimento sob salinidade tem sido atribuída ao estresse osmótico, provocado pela redução do potencial hídrico externo, e ao efeito iônico causado pelo acúmulo de íons nos tecidos vegetais (Munns \& Tester, 2008). O primeiro efeito predomina nas respostas de

Pesq. agropec. bras., Brasília, v.46, n.6, p.586-592, jun. 2011 
curta exposição ao excesso de sais, condição em que as plantas sofrem estresse osmótico e deficiência hídrica resultante da diminuição da absorção de água pelas raízes. A toxicidade iônica, no entanto, pode se desenvolver a longo prazo (Munns \& Tester, 2008) e levar a alterações em uma grande variedade de reações metabólicas.

Entre os vários processos afetados pela salinidade, a redução da fotossíntese está amplamente relatada na literatura (Netondo et al., 2004a, 2004b; Praxedes et al., 2010; Silva et al., 2011) e tem sido atribuída a limitações de origem estomática e não estomática (Netondo et al., 2004b; Munns \& Tester, 2008; Praxedes et al., 2010). Sabe-se que a atividade fotoquímica apresenta resistência ao estresse salino de curta duração (Netondo et al., 2004b), sem redução da eficiência quântica potencial do fotossistema II (Praxedes et al., 2010). Contudo, sob exposição prolongada a sais, quando altas concentrações de íons se acumulam nos tecidos, a atividade fotoquímica também pode ser afetada (Silva et al., 2010).

Em condições de salinidade, o ajuste osmótico pode ser considerado um mecanismo adaptativo que proporciona a manutenção, sob baixos potenciais hídricos, da turgescência e de seus processos dependentes, como abertura estomática, fluxo de água para as células em crescimento e alongamento celular (Silveira et al., 2009). Sob o estresse salino, o ajuste osmótico pode ser alcançado pelo acúmulo de solutos orgânicos, com destaque para a prolina e os açúcares solúveis. Porém, o acúmulo dos sais absorvidos é mais importante, quantitativamente, para o ajuste osmótico da maioria das halófitas e das glicófitas (Silveira et al., 2009). O feijoeiro caupi [Vigna unguiculata (L.) Walp] é considerado uma espécie bem adaptada à salinidade, cuja tolerância tem sido associada, pelo menos em parte, à restrição do acúmulo de $\mathrm{Na}^{+}$nas folhas, que é compartimentalizado no sistema radicular nos estágios iniciais de exposição à salinidade (Cavalcanti et al., 2004).

Sob condições salinas, os sais acumulados nas folhas podem afetar diversos processos fisiológicos das plantas de forma negativa, ao reduzir a fotossíntese, ou positiva, desde que não sejam atingidos níveis tóxicos, pela promoção do ajuste osmótico, que contribui para manutenção da turgescência e do crescimento. No entanto, de acordo com Praxedes et al. (2010), os mecanismos pelos os quais a salinidade afeta a fotossíntese de feijoeiro caupi ainda são desconhecidos. Como a fotossíntese é crucial para a produtividade das plantas, o conhecimento dos mecanismos que conferem resistência à salinidade com relação à eficiência fotossintética é fundamental, especialmente para as plantas cultivadas no semiárido.

O objetivo deste trabalho foi determinar as respostas fotossintéticas e a variação nos teores de carboidratos, íons salinos e prolina em feijoeiro caupi submetido à salinidade.

\section{Material e Métodos}

O experimento foi realizado com a cultivar Vita 7 de feijoeiro caupi. As sementes foram previamente desinfetadas com hipoclorito de sódio $10 \%\left(\mathrm{v} \mathrm{v}^{-1}\right)$ e semeadas em vasos de $2,8 \mathrm{~L}$ contendo substrato sílica:vermiculita (1:1). As plantas foram cultivadas em casa de vegetação, em Campinas, SP (22054'S, $47^{\circ} 05^{\prime} \mathrm{W}$, a $674 \mathrm{~m}$ de altitude), onde a temperatura média do ar variou de 22 a $24^{\circ} \mathrm{C}$, e a máxima radiação fotossinteticamente ativa foi de $1.200 \mu \mathrm{mol} \mathrm{m} \mathrm{m}^{-2} \mathrm{~s}^{-1}$. Cada vaso recebeu solução nutritiva completa de Hoagland \& Arnon (1938) duas vezes por semana ( 0,25 $\mathrm{L}$ por vaso). As plantas foram submetidas à quatro tratamentos, dos 28 aos 35 dias de idade: ausência de $\mathrm{NaCl}$ (controle) e 50, 100 e $200 \mathrm{mmol} \mathrm{L}^{-1}$ de $\mathrm{NaCl}$, cujos respectivos potenciais osmóticos foram $-0,18$, $-0,32,-0,50$ e $-1,12 \mathrm{MPa}$. As soluções salinas foram fornecidas diariamente às plantas por um período de sete dias. Utilizou-se o delineamento experimental de blocos ao acaso, com cinco repetições.

A partir do início do tratamento com as soluções salinas, as plantas foram diariamente transferidas, às 7:00 h, para câmara de crescimento com condições controladas de temperatura do ar $\left(28^{\circ} \mathrm{C}\right)$, radiação fotossinteticamente ativa $\left(\mathrm{Q}=270 \mu \mathrm{mol} \mathrm{m} \mathrm{m}^{-2} \mathrm{~s}^{-1}\right)$ e umidade relativa do ar (70\%). Após 2 horas de aclimatação, foram realizadas as avaliações fisiológicas e, em seguida, as plantas eram levadas de volta para a casa de vegetação. Os folíolos centrais de folhas completamente expandidas foram utilizados para avaliação das características fisiológicas.

Após o início do tratamento, foram realizadas medidas diárias de assimilação líquida de $\mathrm{CO}_{2}\left(\mathrm{P}_{\mathrm{N}}\right)$, de condutância estomática $\left(\mathrm{g}_{\mathrm{s}}\right)$ e da concentração intercelular de $\mathrm{CO}_{2}\left(\mathrm{C}_{\mathrm{I}}\right)$, com um analisador portátil de fotossíntese por radiação infravermelha LCi, ADC (Bioscientific, Hertfordshire, Reino Unido). As medidas foram realizadas com $\mathrm{Q}=1.800 \mu \mathrm{mol} \mathrm{m}^{-2} \mathrm{~s}^{-1}$, fornecida por luzactínica externa. Estimou-se a eficiência aparente 
de carboxilação da Rubisco (ribulose-1,5-bisfosfato carboxilase/oxigenase) pela razão $\mathrm{P}_{\mathrm{N}} / \mathrm{C}_{\mathrm{I}}$ (Ribeiro et al., 2009). Os resultados medidos da fotossíntese diária foram integrados, para calcular a redução de cada tratamento em comparação ao controle.

As medidas da emissão de fluorescência da clorofila $a$ foram realizadas com fluorômetro modulado FMS1, (Hansatech, Norfolk, Reino Unido), pelo método do pulso de saturação $\left(\lambda<710 \mathrm{~nm}, \mathrm{Q} \sim 10.000 \mu \mathrm{mol} \mathrm{m}^{-2} \mathrm{~s}^{-1}\right)$. Foram avaliados a eficiência quântica potencial $\left(\mathrm{F}_{\mathrm{V}} / \mathrm{F}_{\mathrm{M}}\right)$ e efetiva $\left(\Delta F / F_{M}\right.$ ') do fotossistema II, e os coeficientes de extinção fotoquímica (qP) e não fotoquímica (NPQ) da fluorescência, de acordo com Ribeiro et al. (2009). As medidas realizadas na presença de luz foram feitas em folhas adaptadas às condições prevalecentes na câmara de crescimento, após exposição, por $10 \mathrm{~s}$, a um feixe de luz de $270 \mu \mathrm{mol} \mathrm{m}^{-2} \mathrm{~s}^{-1}$ fornecido pela própria fibra óptica do aparelho. As medidas de atividade fotoquímica e das relações hídricas foram realizadas no sétimo dia após o início do tratamento salino.

O potencial total da água na folha foi avaliado com câmara de pressão Soilmoisture 3005, (Soilmoisture Equipament Corporation, Santa Barbara, CA, EUA), e o potencial osmótico na turgescência plena com o uso de câmaras psicrométricas C-52, (Wescor Inc., Logan, Utah, EUA) conectadas a um microvoltímetro de ponto de orvalho HR-33T, (Wescor Inc., Logan, Utah, EUA). Neste caso, as folhas foram coletadas com o pecíolo cortado sob água destilada, para evitar a entrada de ar no xilema. A porção cortada foi mantida submersa em água destilada em câmara úmida no escuro por cerca de 20 horas, quando os discos foliares foram retirados e imediatamente congelados em nitrogênio líquido. Após o descongelamento, os discos foram inseridos nas câmaras psicrométricas, e o potencial osmótico foi determinado após 30 min de equilíbrio.

No sétimo dia do tratamento salino, foram coletadas amostras de tecido foliar, as quais foram secadas em estufa $\left(70^{\circ} \mathrm{C}\right)$ por 36 horas e moídas para quantificação de sódio (Bataglia et al., 1983) e cloreto (Walinga et al., 1989). Outras amostras de tecido foliar foram coletadas e imediatamente congeladas em nitrogênio líquido, para posterior determinação do conteúdo de carboidratos e prolina, após liofilização. A solução utilizada para quantificar sacarose, açúcares solúveis e prolina foi obtida a partir de extração com metanol, clorofórmio e água (MCW, 12:5:3 $\mathrm{v} \mathrm{v}^{-1}$ ), segundo Bieleski \& Turner (1966). As dosagens de açúcares solúveis foram determinadas pelo método da antrona (McReady et al., 1950) e as de sacarose de acordo com Handel (1968). A quantificação de amido foi realizada segundo o método descrito por Yemm \& Willis (1954), e as dosagens foram feitas pelo método da antrona (McReady et al., 1950). A dosagem de prolina foi determinada conforme Rena \& Masciotti (1976).

Osresultados foram submetidos à análise de variância, e as médias dos tratamentos foram comparadas pelo teste de Tukey, a 5\% de probabilidade.

\section{Resultados e Discussão}

Houve diminuição do potencial total da água nas folhas de plantas de feijoeiro caupi apenas no tratamento com $200 \mathrm{mmol} \mathrm{L}^{-1}$ de $\mathrm{NaCl}$ (Tabela 1). $\mathrm{O}$ potencial osmótico em turgescência plena, apesar de ter apresentado tendência de diminuição com as concentrações crescentes de $\mathrm{NaCl}$, diminuiu significativamente somente no tratamento com maior salinidade (200 mmol L-1 de $\mathrm{NaCl})$, o que indica acúmulo de solutos nos tecidos foliares apenas neste tratamento. De fato, além de acúmulo do íon cloreto $\left(\mathrm{Cl}^{-}\right)$, as plantas do tratamento com $200 \mathrm{mmol} \mathrm{L}^{-1}$ de $\mathrm{NaCl}$ apresentaram as maiores concentrações de prolina e de açúcares solúveis. Contudo, as concentrações observadas foram quantitativamente muito baixas para causar ajuste osmótico, como relatado por Silva et al. (2009), em trabalho com pinhão manso. Portanto, a redução observada no potencial osmótico não esteve relacionada a um efetivo ajustamento osmótico, o que

Tabela 1. Potencial hídrico total $\left(\Psi_{t}\right)$, potencial osmótico $\left(\Psi_{0}\right)$ e concentração de $\mathrm{Na}^{+}, \mathrm{Cl}^{-}$, prolina, açúcares solúveis (AS), sacarose e amido na folha de feijoeiro caupi, após sete dias submetido à doses crescentes de $\mathrm{NaCl}$ no substrato de crescimento $^{(1)}$.

\begin{tabular}{lcccc}
\hline Variável & \multicolumn{4}{c}{ Tratamento $\left(\mathrm{mmol} \mathrm{L}^{-1} \mathrm{de} \mathrm{NaCl}\right)$} \\
\cline { 2 - 5 } & 0 & 50 & 100 & 200 \\
\hline$\Psi_{\mathrm{t}}(\mathrm{MPa})$ & $-0,39 \mathrm{~b}$ & $-0,38 \mathrm{~b}$ & $-0,50 \mathrm{~b}$ & $-0,72 \mathrm{a}$ \\
$\Psi_{0}(\mathrm{MPa})$ & $-0,61 \mathrm{~b}$ & $-0,66 \mathrm{ab}$ & $-0,68 \mathrm{ab}$ & $-0,73 \mathrm{a}$ \\
$\mathrm{Na}^{+}(\mathrm{mmol} \mathrm{kg} \mathrm{ke} \mathrm{MS})^{-1}$ & $20,5 \mathrm{a}$ & $27,8 \mathrm{a}$ & $30,5 \mathrm{a}$ & $22,0 \mathrm{a}$ \\
$\mathrm{Cl}^{-}(\mathrm{mmol} \mathrm{kg} \mathrm{de} \mathrm{MS})^{24,6 \mathrm{c}}$ & $314,1 \mathrm{~b}$ & $345,0 \mathrm{~b}$ & $517,7 \mathrm{a}$ \\
Prolina $\left(\mathrm{mmol} \mathrm{kg}^{-1} \mathrm{de} \mathrm{MS}\right)$ & $1,09 \mathrm{c}$ & $1,30 \mathrm{c}$ & $1,85 \mathrm{~b}$ & $4,17 \mathrm{a}$ \\
$\mathrm{AS}\left(\mathrm{g} \mathrm{kg}^{-1} \mathrm{de} \mathrm{glicose}^{-1}\right)$ & $32,1 \mathrm{~b}$ & $35,6 \mathrm{~b}$ & $45,6 \mathrm{a}$ & $44,6 \mathrm{a}$ \\
Sacarose $\left(\mathrm{g} \mathrm{kg}^{-1}\right.$ de glicose $)$ & $20,1 \mathrm{~b}$ & $19,6 \mathrm{~b}$ & $28,0 \mathrm{a}$ & $26,2 \mathrm{a}$ \\
Amido $\left(\mathrm{g} \mathrm{kg}^{-1}\right.$ de glicose $)$ & $61,0 \mathrm{a}$ & $60,9 \mathrm{a}$ & $74,6 \mathrm{a}$ & $35,6 \mathrm{~b}$ \\
\hline
\end{tabular}

${ }^{(1)}$ Médias seguidas de letras iguais, nas linhas, não diferem entre si pelo teste de Tukey, a 5\% de probabilidade. MS, massa seca. 
confirma o observado por Silveira et al. (2003), em plantas de feijão-caupi expostas ao estresse hídrico.

A concentração de $\mathrm{Na}^{+}$nas folhas, após sete dias de tratamento salino, não variou significativamente entre os tratamentos (Tabela 1). Esse resultado indica a presença de um mecanismo ativo de exclusão do elemento nas folhas, que o teria retido nas raízes e bloqueado o transporte para a parte aérea das plantas, como previamente relatado por Cavalcanti et al. (2004). De acordo com Praxedes et al. (2010), o mecanismo de restrição do movimento de $\mathrm{Na}^{+}$para a parte aérea das plantas é uma característica associada à tolerância do gênero Vigna à salinidade.

Houve aumento na concentração foliar de $\mathrm{Cl}^{-}$com o aumento da disponibilidade de $\mathrm{NaCl}$ no substrato (Tabela 1). Dutra et al. (2011) demonstraram que o acúmulo de $\mathrm{Cl}^{-}$em folhas de feijoeiro caupi é maior do que o de $\mathrm{Na}^{+}$, e que o inverso ocorre nas raízes. Aparentemente, os mecanismos de exclusão de $\mathrm{Cl}^{-}$nas folhas são menos efetivos, possivelmente por um maior transporte via apoplasto (Neocleous \& Vasilakakis, 2007; Ferreira-Silva et al., 2010), em comparação ao $\mathrm{Na}^{+}$. Em algumas espécies, o acúmulo excessivo de $\mathrm{Cl}^{-}$pode causar toxicidade (Neocleous \& Vasilakakis, 2007), o que justificaria o decréscimo na fotossíntese nos níveis mais elevados de $\mathrm{NaCl}$ (Figuras 1 e 2).

A imposição do estresse salino causou um padrão nítido de diminuição da assimilação líquida de $\mathrm{CO}_{2}\left(\mathrm{P}_{\mathrm{N}}\right)$ ao longo do tempo, em função da crescente concentração de sal na solução nutritiva (Figura 1 A). A concentração de $50 \mathrm{mmol} \mathrm{L}^{-1}$ afetou negativa e significativamente a $\mathrm{P}_{\mathrm{N}}$ somente no sétimo dia de tratamento, enquanto, nos tratamentos com 100 e $200 \mathrm{mmol} \mathrm{L}^{-1}$ de $\mathrm{NaCl}$, as reduções ocorreram, respectivamente, a partir do terceiro e do segundo dia da imposição do estresse. O efeito da salinidade na fotossíntese, no decorrer do experimento, foi marcante, com redução acumulada, durante os sete dias de tratamento, de 6,5, 21,3 e 47,1\%, sob 50, 100 e $200 \mathrm{mmol} \mathrm{L}^{-1}$ de $\mathrm{NaCl}$, respectivamente (Figura $1 \mathrm{~B}$ ).

O padrão nitidamente decrescente da resposta fotossintética ao aumento da concentração de $\mathrm{NaCl}$ no substrato também foi observado para condutância estomática $\left(\mathrm{g}_{\mathrm{s}}\right)$ e eficiência de carboxilação da Rubisco, estimada pela razão $\mathrm{P}_{\mathrm{N}} / \mathrm{C}_{\mathrm{I}}$ (Figura 2). Esses resultados estão de acordo com os obtidos por Bethke \& Drew (1992), Netondo et al. (2004b), Zhao et al. (2007) e Youssef \& Awad (2008).
$\mathrm{AP}_{\mathrm{N}} / \mathrm{C}_{\mathrm{I}}$ decresceu linearmente com o acúmulo de $\mathrm{Cl}^{-}$ no tecido foliar (Figura $3 \mathrm{~A}$ ), o que pode ser explicado pelo efeito tóxico de concentrações crescentes de $\mathrm{Cl}^{-}$ nas folhas, já relatado em outras espécies (Bethke \& Drew, 1992; Netondo et al., 2004; Neocleous \& Vasilakakis, 2007). No entanto, a ausência de relação entre a $\mathrm{P}_{\mathrm{N}} / \mathrm{C}_{\mathrm{I}}$ e o teor de $\mathrm{Na}^{+}$não confirma os resultados de outros trabalhos (Bethke \& Drew, 1992; Netondo et al., 2004b; Zhao et al., 2007). É provável que a redução em $\mathrm{P}_{\mathrm{N}} / \mathrm{C}_{\mathrm{I}}$ esteja associada à diminuição de $\mathrm{C}_{\mathrm{I}}$

\begin{tabular}{|c|c|}
\hline - Controle & $-\mathrm{O}-50 \mathrm{mmol} \mathrm{L}^{-1} \mathrm{NaCl}$ \\
\hline$\triangle 100 \mathrm{mmol} \mathrm{L}^{-1} \mathrm{NaCl}$ & $\nabla 200 \mathrm{mmol} \mathrm{L}^{-1} \mathrm{NaCl}$ \\
\hline
\end{tabular}
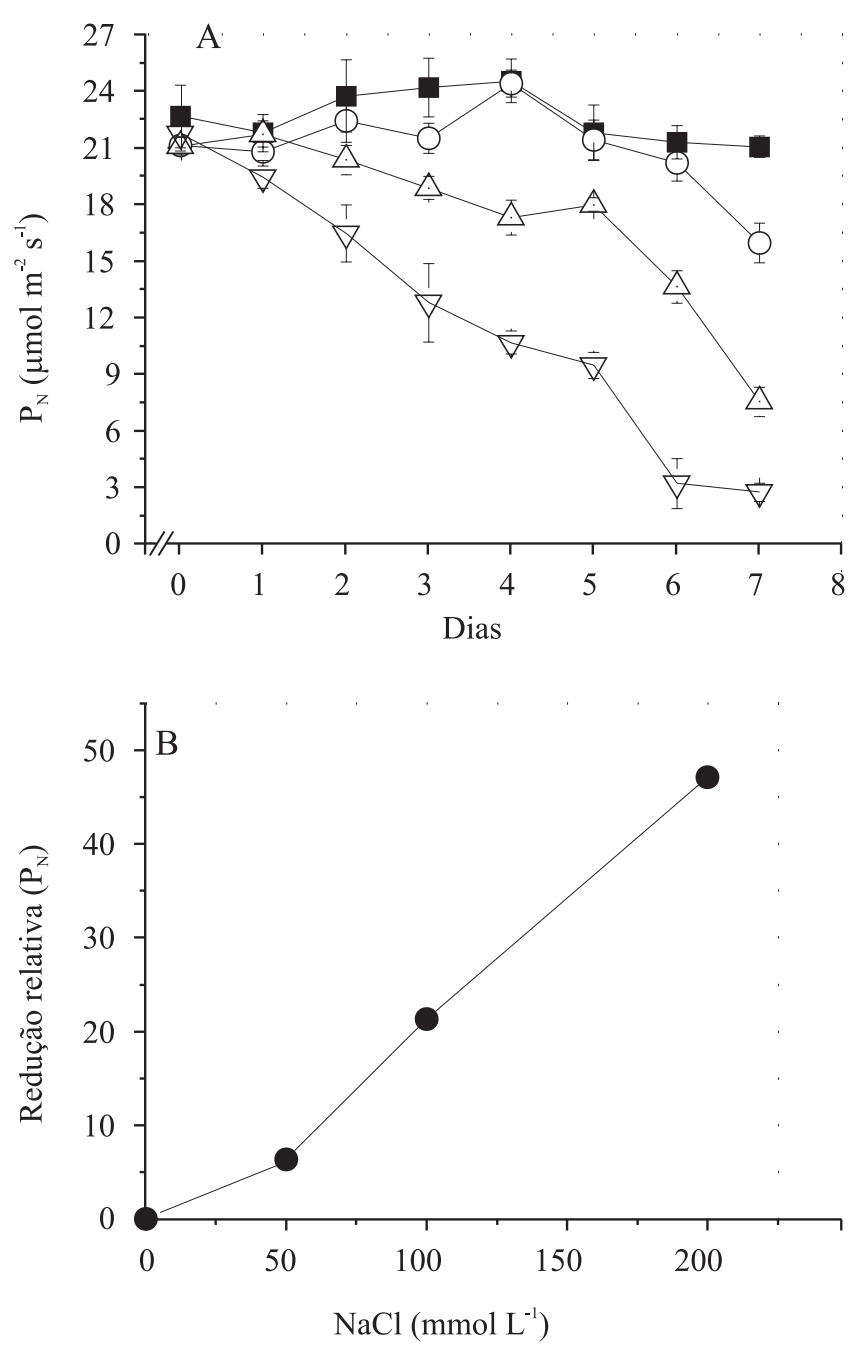

Figura 1. Variação da assimilação líquida de $\mathrm{CO}_{2}\left(\mathrm{P}_{\mathrm{N}}\right)$ em feijoeiro caupi, em função do aumento da concentração de $\mathrm{NaCl}$ no substrato de crescimento e do tempo de tratamento (A); e redução relativa da $\mathrm{P}_{\mathrm{N}}$ integrada durante os dias de tratamento (B). Barras indicam desvio-padrão. 
(Figura $2 \mathrm{~A}$ e B) e, portanto, à menor disponibilidade de $\mathrm{CO}_{2}$ como substrato para fotossíntese (Bethke \& Drew, 1992). Esse decréscimo em $C_{I}$ pode ser atribuído às menores condutâncias estomáticas, uma resposta comum das plantas ao estresse salino (Praxedes et al., 2010; Silva et al., 2011). Os resultados obtidos no tratamento com $100 \mathrm{mmol} \mathrm{L}^{-1}$ de $\mathrm{NaCl}$, com redução significativa de $\mathrm{P}_{\mathrm{N}}$ e de $\mathrm{g}_{\mathrm{s}}$, acompanhada de variação não significativa do potencial hídrico foliar $\left(\Psi_{\mathrm{W}}\right)$ (Figura $3 \mathrm{~B}$, Tabela 1), indicam a ocorrência de sinalização química entre raízes e parte aérea que mediariam o fechamento estomático. Zeng et al. (2001) verificaram que a transpiração e a $g_{s}$ diminuem com a salinidade na rizosfera, provavelmente pela queda do potencial da água nas raízes ou pelo transporte de ácido abscísico para as folhas (James et al., 2002). É possível,
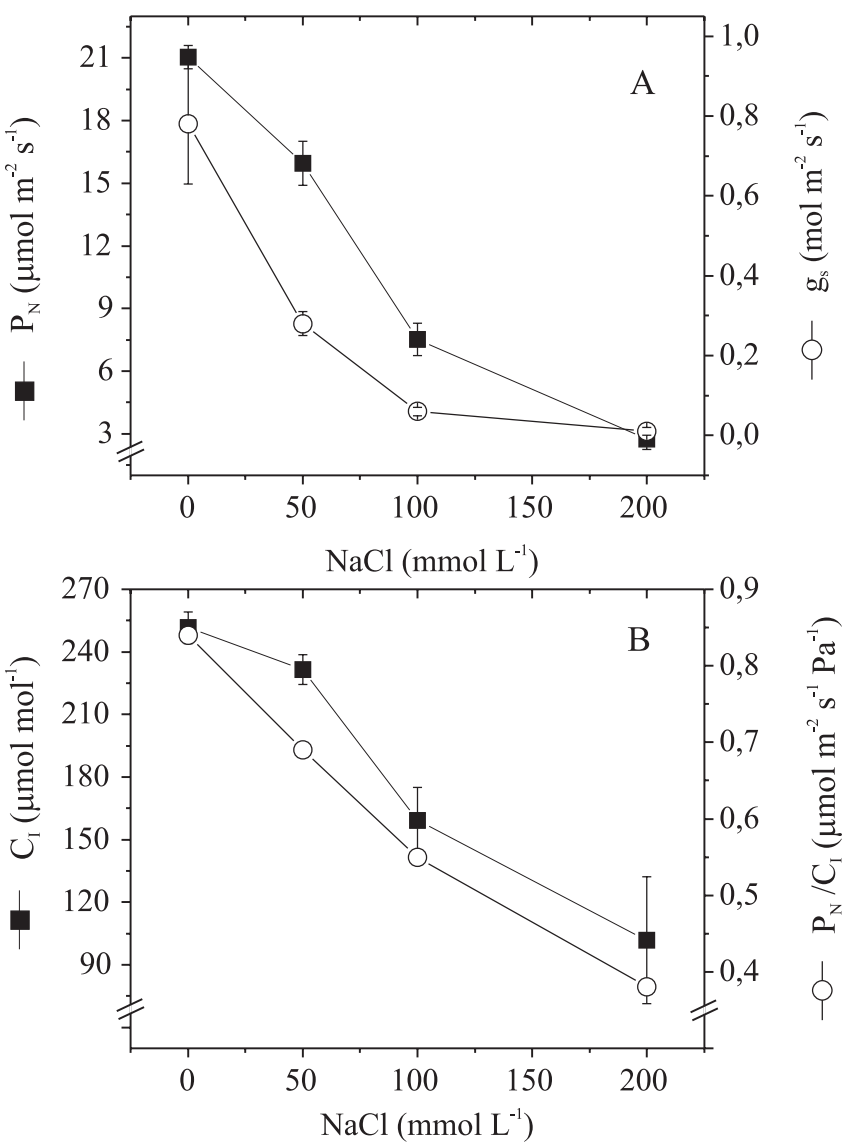

Figura 2. Variação da assimilação de $\mathrm{CO}_{2}\left(\mathrm{P}_{\mathrm{N}}\right)$ e da condutância estomática $\left(\mathrm{g}_{\mathrm{s}}\right)(\mathrm{A}) \mathrm{e}$, da concentração interna de $\mathrm{CO}_{2}(\mathrm{CI})$ e da eficiência aparente de carboxilação da ribulose-1,5-bisfosfato carboxilase/oxigenase $\left(\mathrm{P}_{N} / \mathrm{CI}\right)(\mathrm{B})$, em função do aumento da concentração de $\mathrm{NaCl}$ no substrato de crescimento, após sete dias de tratamento. Barras indicam desvio-padrão. também, que o acúmulo do íon $\mathrm{Cl}^{-}$nas folhas tenha contribuído para a resposta de fechamento estomático, dada as diminuições observadas em $\mathrm{g}_{\mathrm{s}}$ pelo aumento da concentração foliar deste íon (Figura $3 \mathrm{~A}$ ).

A eficiência quântica potencial do fotossistema II $\left(\mathrm{F}_{\mathrm{V}} /\right.$ $F_{M}$ ) variou entre 0,82 e 0,85 (Figura 4), o que evidencia uma grande resistência da atividade fotoquímica do feijoeiro caupi ao estresse salino. Na literatura, há estudos que apontam tanto a alta (Netondo et al., 2004b) como a baixa (Misra et al., 2001; Moradi \& Ismail, 2007; Silva et al., 2011) sensibilidade de $F_{V} / F_{M}$
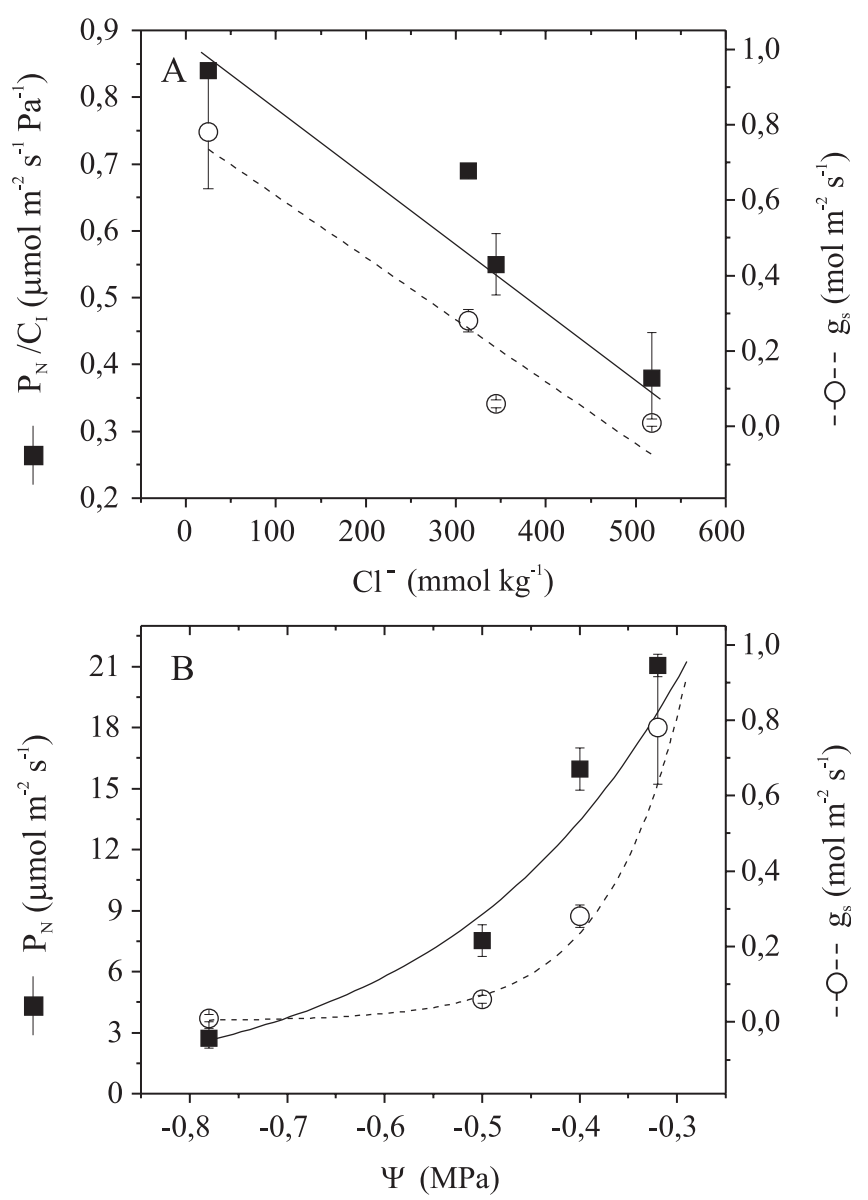

Figura 3. Variação da eficiência aparente de carboxilação da ribulose-1,5-bisfosfato carboxilase/oxigenase $\left(\mathrm{P}_{\mathrm{N}} / \mathrm{C}_{\mathrm{I}}=\right.$ $\left.-0,0009\left[\mathrm{Cl}^{-}\right]+0,89 ; \mathrm{R}^{2}=0,91, \mathrm{p}<0,05\right)$ e da condutância estomática $\left(\mathrm{g}_{\mathrm{s}}=0,776-0,00164\left[\mathrm{Cl}^{-}\right] ; \mathrm{R}^{2}=0,91, \mathrm{p}<0,05\right)$, em função da concentração de $\mathrm{Cl}^{-}$na folha (A); e variação da assimilação de $\mathrm{CO}_{2}\left(\mathrm{P}_{\mathrm{N}}=70,77 \exp (\Psi / 0,24)-0,249\right.$; $\left.\mathrm{R}^{2}=0,96, \mathrm{p}<0,05\right)$ e de $\mathrm{g}_{\mathrm{s}}\left(\mathrm{g}_{\mathrm{s}}=33,71 \exp (\Psi / 0,08)+0,003\right.$; $\left.\mathrm{R}^{2}=0,95, \mathrm{p}<0,05\right)$, em função do potencial da água na folha $(\Psi)$, em feijoeiro caupi após sete dias de tratamento com doses crescentes de $\mathrm{NaCl}$ no substrato de crescimento (B). Barras indicam desvio-padrão. 
ao aumento de $\mathrm{NaCl}$ no substrato, o que indica que o efeito da salinidade pode ser específico para as formas de imposição do estresse salino e para as condições de crescimento da planta.

Em relação à atividade fotoquímica sob pressão energética, as maiores alterações foram notadas no tratamento com $200 \mathrm{mmol} \mathrm{L}^{-1}$ de $\mathrm{NaCl}$ (Figura 4). $\mathrm{Na}$ comparação com as plantas controle, foram observados pequenos decréscimos na eficiência efetiva do fotossistema II $\left(\Delta F / F_{M}{ }^{\prime}\right)$ e do coeficiente de "quenching" fotoquímico (qP), além de pequenos aumentos do "quenching" não fotoquímico (NPQ). Esses aumentos, associados aos mecanismos de dissipação não fotoquímica da energia, são causados pelas reduções de $\Delta \mathrm{F} / \mathrm{F}_{\mathrm{M}}$ ' e de $\mathrm{qP}$, esperadas em virtude
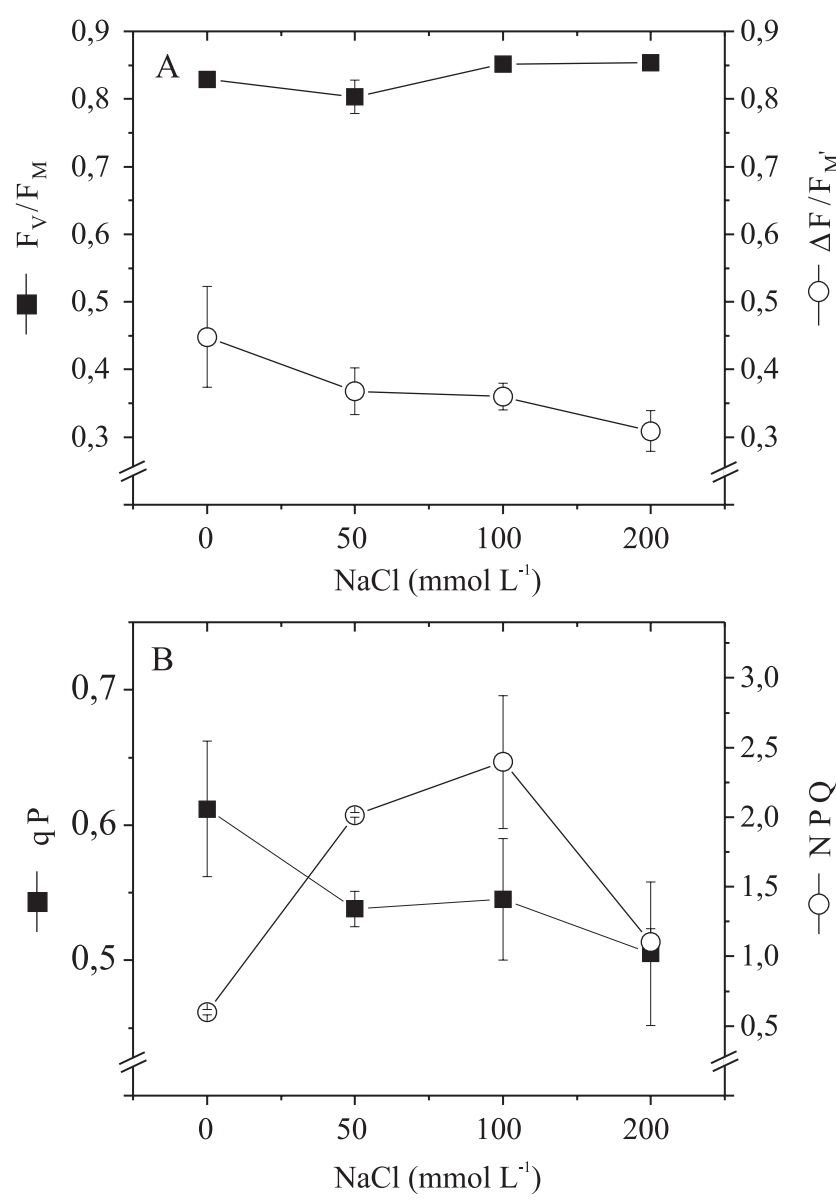

Figura 4. Variação da eficiência quântica potencial $\left(\mathrm{F}_{\mathrm{V}} / \mathrm{F}_{\mathrm{M}}\right)$ e efetiva $\left(\Delta F / F_{M}{ }^{\prime}\right)$ do fotossistema II $(A)$ e do coeficiente de extinção fotoquímica (qP) e não fotoquímica (NPQ) da fluorescência, em feijoeiro caupi após sete dias de tratamento com doses crescentes de $\mathrm{NaCl}$ no substrato de crescimento (B). Barras indicam desvio-padrão. da redução de $\mathrm{P}_{\mathrm{N}}$ pelo estresse salino (Silva et al., 2011). Em geral, a atividade fotoquímica pareceu não limitar a fixação de $\mathrm{CO}_{2}$ em feijoeiro caupi sob estresse salino, e as respostas fotoquímicas podem ser consideradas como aclimatação no sentido de reduzir o excesso de excitação nos fotossistemas.

Quanto aos produtos finais da fotossíntese, houve redução do teor de amido apenas no tratamento com $200 \mathrm{mmol} \mathrm{L}^{-1}$ de $\mathrm{NaCl}$ (Tabela 1), possivelmente pela acentuada redução na fotossíntese ou como resultado de sua hidrólise, já que os valores de sacarose e açúcares solúveis encontraram-se aumentados nos tratamentos mais severos. É provável que a hidrólise do amido tenha ocorrido como consequência da limitação da disponibilidade de açúcares para o metabolismo celular, em decorrência das restrições à assimilação de $\mathrm{CO}_{2}$ provocada pelo estresse salino.

\section{Conclusões}

1. As reduções na assimilação líquida de $\mathrm{CO}_{2}$, em feijoeiro caupi sob estresse salino, são ocasionadas pela redução da abertura estomática, acompanhada pelo decréscimo da eficiência aparente de carboxilação.

2. O íon cloreto tem papel determinante na redução da fotossíntese em feijoeiro caupi submetido à salinidade.

3. O feijoeiro caupi apresenta características fisiológicas favoráveis à manutenção do processo fotossintético sob curta exposição à salinidade, especialmente quanto à atividade fotoquímica.

4. A exclusão foliar de $\mathrm{Na}^{+}$e a manutenção da disponibilidade de carboidratos solúveis, mesmo sob altas concentrações de $\mathrm{NaCl}$, contribuem para a resistência ao estresse salino.

\section{Agradecimentos}

Ao Conselho Nacional de Desenvolvimento Científico e Tecnológico, pelo apoio financeiro.

\section{Referências}

BATAGLIA, O.C.; FURLANI, A.M.C.; TEIXEIRA, J.P.F.; FURLANI, P.R.; GALLO, J.R. Métodos de análise química de plantas. Campinas: Instituto Agronômico, 1983. 48p. (IAC. Boletim técnico, 78).

BETHKE, P.C.; DREW, M.C. Stomatal and nonstomatal components to inhibition of photosynthesis in leaves of Capsicum annuum during progressive exposure to $\mathrm{NaCl}$ salinity. Plant Physiology, v.99, p.219-226, 1992. 
BIELESKI, R.L.; TURNER, A. Separation and estimation of amino acids in crude plant extracts by thin-layer electrophoresis and chromatography. Analytical Biochemistry, v.17, p.278-293, 1966.

CAVALCANTI, F.R.; OLIVEIRA, J.T.A.; MARTINS-MIRANDA, A.S.; VIÉGAS, R.A.; SILVEIRA, J.A.G. Superoxide dismutase, catalase and peroxidase activities do not confer protection against oxidative damage in salt-stressed cowpea leaves. New Phytologist, v.163, p.563-571, 2004.

DUTRA, A.T.B.; SILVA, E.N.; RODRIGUES, C.R.F.; VIEIRA, S.A.; ARAGÃO, R.M.; SILVEIRA, J.A.G. Temperaturas elevadas afetam a distribuição de íons em plantas de feijão Caupi pré-tratadas com $\mathrm{NaCl}$. Revista Brasileira de Engenharia Agrícola e Ambiental, v.15, p.403-409, 2011.

FERREIRA-SILVA, S.L.; SILVA, E.N.; CARVALHO, F.E.L.; LIMA, C.S.; ALVES, F.A.L.; SILVEIRA, J.A.G. Physiological alterations modulated by rootstock and scion combination in cashew under salinity. Scientia Horticulturae, v.127, p.39-45, 2010.

HANDEL, E. van. Direct microdetermination of sucrose. Analytical Biochemistry, v.22, p.280-283, 1968.

HOAGLAND, D.R.; ARNON, D.I. The water-culture method for growing plants without soil. Berkeley: University of California, 1938. 39p.

JAMES, R.A.; RIVELLI, A.R.; MUNNS, R.; VON CAEMMERER, $\mathrm{S}$. Factors affecting $\mathrm{CO}_{2}$ assimilation, leaf injury and growth in salt-stressed durum wheat. Functional Plant Biology, v.29, p.1393-1403, 2002.

MCREADY, R.M.; GUGGOLZ, J.; SILVEIRA, Y.; OWENS, H.S. Determination of starch and amylose in vegetables. Analytical Chemistry, v.22, p.1156-1158, 1950.

MISRA, A.N.; SRIVASTAVA, A.; STRASSER, R.J. Utilization of fast chlorophyll fluorescence technique in assessing the salt/ion sensitivity of mung bean and Brassica seedlings (Hordeum vulgare L.). Journal of Plant Physiology, v.158, p.1173-1181, 2001.

MORADI, F.; ISMAIL, A.M. Responses of photosynthesis, chlorophyll fluorescence and ROS-scavenging systems to salt stress during seedling and reproductive stages in rice. Annals of Botany, v.99, p.1161-1173, 2007.

MUNNS, R.; TESTER, M. Mechanisms of salinity tolerance. Annual Review of Plant Biology, v.59, p.651-681, 2008.

NEOCLEOUS, D.; VASILAKAKIS, M. Effects of $\mathrm{NaCl}$ stress on red raspberry (Rubus idaeus L. 'Autumn Bliss'). Scientia Horticulturae, v.112, p.282-289, 2007.

NETONDO, G.W.; ONYANGO, J.C.; BECK, E. Sorghum and salinity: I. Response of growth, water relations, and ion accumulation to $\mathrm{NaCl}$ salinity. Crop Science, v.44, p.797-805, 2004a.

NETONDO, G.W.; ONYANGO, J.C.; BECK, E. Sorghum and salinity: II. Gas exchange and chlorophyll fluorescence of sorghum under salt stress. Crop Science, v.44, p.806-811, 2004 b.

PRAXEDES, S.C.; LACERDA, C.F. de; DAMATTA, F.M.; PRISCO, J.T.; GOMES-FILHO, E. Salt tolerance is associated with differences in ion accumulation, biomass allocation and photosynthesis in cowpea cultivars. Journal of Agronomy and Crop Science, v.196, p.193-204, 2010.

RENA, A.B.; MASCIOTTI, G.Z. Efeito do déficit hídrico sobre o metabolismo do nitrogênio e o crescimento de quatro cultivares de feijão (Phaseolus vulgaris L.). Revista Ceres, v.23, p.288-301, 1976.

RIBEIRO, R.V.; MACHADO, E.C.; SANTOS, M.G.; OLIVEIRA, R.F. Photosynthesis and water relations of well-watered orange plants as affected by winter and summer conditions. Photosynthetica, v.47, p.215-222, 2009.

SILVA, E.N. da; RIBEIRO, R.V.; FERREIRA-SILVA, S.L.; VIÉGAS, R.A.; SILVEIRA, J.A.G. Comparative effects of salinity and water stress on photosynthesis, water relations and growth of Jatropha curcas plants. Journal of Arid Environments, v.74, p.1130-1137, 2010.

SILVA, E.N. da; RIBEIRO, R.V.; FERREIRA-SILVA, S.L.; VIÉGAS, R.A.; SILVEIRA, J.A.G. Salt stress induced damages on the photosynthesis of physic nut young plants. Scientia Agricola, v.68, p.62-68, 2011.

SILVA, E.N. da; SILVEIRA, J.A.G.; FERNANDES, C.R.R.; DUTRA, A.T.B.; ARAGÃO, R.M. de. Acúmulo de íons e crescimento de pinhão-manso sob diferentes níveis de salinidade. Revista Ciência Agronômica, v.40, p.22-29, 2009.

SILVEIRA, J.A.G.; ARAÚJO, S.A.M.; LIMA, J.P.M.S.; VIÉGAS, R.A. Roots and leaves display contrasting osmotic adjustment mechanisms in response to $\mathrm{NaCl}$-salinity in Atriplex numularia. Environmental and Experimental Botany, v.66, p.1-8, 2009.

SILVEIRA, J.A.G.; COSTA, R.C.L.; VIÉGAS, R.A.; OLIVEIRA, J.T.A.; FIGUEREDO; M.V.B. N-compound accumulation and carbohydrate shortage on $\mathrm{N}_{2}$ fixation in drought-stressed and rewatered cowpea plants. Spanish Journal of Agricultural Research, v.1, p.65-75, 2003.

WALINGA, I.; VAN VARK, W.; HOUBA, V.J.G.; VAN DER LEE, J.J. Plant analysis procedures. Wagenigen: Agricultural University, 1989. 263p. (Soil and plant analysis, part 7).

YEMM, E.W.; WILLIS, A.J. The estimation of carbohydrates in plant extracts by anthrone. Biochemical Journal, v.57, p.508-514, 1954.

YOUSSEF, T.; AWAD, M.A. Mechanisms of enhancing photosynthetic gas exchange in date palm seedlings (Phoenix dactylifera L.) under salinity stress by a 5-aminolevulinic acid-based fertilizer. Journal of Plant Growth Regulation, v.27, p.1-9, 2008.

ZENG, L.; SHANNON, M.C.; LESCH, S.M. Timing of salinity stress affects rice growth and yield components. Agriculture and Water Management, v.48, p.191-206, 2001.

ZHAO, G.Q.; MA, B.L.; REN, C.Z. Growth, gas exchange, chlorophyll fluorescence, and ion content of naked oat in response to salinity. Crop Science, v.47, p.123-131, 2007.

Recebido em 19 de dezembro de 2010 e aprovado em 27 de maio de 2011

Pesq. agropec. bras., Brasília, v.46, n.6, p.586-592, jun. 2011 